\title{
Association of the nutrient profile system underlying the Nutri-Score front-of-package label with oral health: preliminary evidence from a population-based sample
}

\begin{abstract}
The 2017 implementation in France of the front-of-package nutrition label known as Nutri-Score is a major public health strategy to help individuals make healthier food choices at the point of purchase and thus help reduce chronic disease risk on the population level. The algorithm behind the Nutri-Score is intended to reflect the overall nutritional quality of food and is based on the one developed by the British Food Standards Agency Nutrient Profiling System, following a slight modification (FSAm-NPS) in accordance with French dietary guidelines. Prior research has focused on its association with various physical health outcomes (weight gain, metabolic syndrome, cancer incidence, etc.), yet no studies have explored the link with oral health indicators. We analyzed the cross-sectional association of the FSAm-NPS with oral health in a population-based sample of 33,231 men and women enrolled in the NutriNet-Santé cohort. Oral health (main dependent variable) was assessed in 2016 with the General Oral Health Assessment Index (GOHAI). It consists of 12 items scored on a 5-point Likert scale (maximum score $=60$ ) with lower scores corresponding to poorer oral health with a detrimental impact on quality of life. FSAm-NPS (main independent variable) was calculated on the basis of 3 non-consecutive 24-h dietary records, following established methodology (score range: -15 to +40 , with lower scores corresponding to higher nutritional quality). Age-specific associations (cutoff $=60 \mathrm{y}$ ) were explored via multivariable linear regression models. Mean GOHAI score in the sample was $53.8 \pm 5.5$ (range: 19 to 60) and mean FSAm-NPS was $6.0 \pm 2.2$ (range: -6.7 to +15.5 ). The unadjusted and the age- and sex-adjusted models were significant only in the younger age group (18-59 y). The fully-adjusted model showed modest yet significant associations between overall nutritional quality and oral health in younger and older participants, with the strength of the model being more pronounced in the former compared with the latter age group $(F$ value: 28.5 vs. 6.3 , both $p<0.0001$ ). Overall, consuming food with a higher FSAm-NPS score (corresponding to lower nutritional quality) was associated with higher risk of oral health problems. Future longitudinal studies are needed to confirm the observed associations. On the public health policy level, the findings support implementation of the Nutri-Score on pre-packaged food in order to steer consumer choice toward nutritional profiles that are favorable not only for chronic disease prevention but also for oral health.
\end{abstract}

\section{Conflict of Interest}

There is no conflict of interest. 